\title{
A Reaction-Controlled Diffusion Model for the Lithiation of Silicon in Lithium-Ion Batteries
}

\author{
Xiaoxuan Zhang ${ }^{\mathrm{a}}$, Seok Woo Lee ${ }^{\mathrm{b}}$, Hyun-Wook Lee ${ }^{\mathrm{c}}$, Yi Cui ${ }^{\mathrm{c}, \mathrm{d}}$, Christian Linder ${ }^{\mathrm{a}, *}$ \\ ${ }^{a}$ Department of Civil and Environmental Engineering, Stanford University, Stanford, California, 94305, USA \\ ${ }^{b}$ Geballe Laboratory for Advanced Materials, Stanford University, Stanford, California 94305, USA \\ ${ }^{c}$ Department of Materials Science and Engineering, Stanford University, Stanford, California 94305, USA \\ ${ }^{d}$ Stanford Institute for Materials and Energy Sciences, SLAC National Accelerator Laboratory, Menlo Park, California 94025, USA
}

\begin{abstract}
Silicon is considered as a promising anode material for lithium ion batteries. Despite the great attention on Si anode materials, a consistent description of the diffusion and reaction mechanism at the reaction front of crystalline silicon, amorphous silicon, and delithiated amorphous silicon has not yet been proposed. To better understand those mechanisms, a new reaction-controlled diffusion formulation is proposed. The new formulation makes use of the bond-breaking energy barrier $E_{0}$ as the key physical quantity. With the consideration of different values of $E_{0}$, the two-phase diffusion during initial lithiation of both crystalline $\mathrm{Si}$ and amorphous Si can be well represented with an evident reaction front. In addition, by varying $E_{0}$, the one phase lithiation of amorphous $\mathrm{Si}$, obtained after the delithiation process, can be captured with the new formulation. The effect of deformation, hydrostatic pressure at the reaction front, and $\mathrm{Li}$ concentration level on the reaction front velocity is taken into account in the proposed model. Numerical simulations are provided to support the model.
\end{abstract}

\section{(c) 2015 Published by Elsevier Ltd.}

Keywords:

reaction-controlled diffusion, bond-breaking energy barrier, hydrostatic pressure effect, silicon electrode, lithium-ion batteries

\section{Introduction}

Lithium-ion batteries (LIBs) have become very important energy storage devices due to their high energy density and high average voltage. LIBs with higher energy storage density and longer cycle life are persistently demanded, especially from the emerging electric vehicle industry [1]. Silicon ( $\mathrm{Si}$ ) is a very attractive anode material for a maximum theoretical specific capacity as high as $4200 \mathrm{mAhg}^{-1}$ and its abundance on earth [2]. However, enormous volume changes ( $\sim 310 \%$ at full lithiation) caused by the insertion and extraction of Lithium (Li) in Si [3] lead to electrode pulverization and capacity loss [2]. Even though nanotechnology [4] has shed lights on the practical application of $\mathrm{Si}$ as the anode material, a thorough understanding of the complicated electro-chemo-mechanical problem involved in the (de)lithiation process of $\mathrm{Si}$ is still missing. In particular, further advanced nanomaterial designs, such as those making use of double-walled Si tubes [5], Si-C yolk-shell and pomegranate [6, 7], or nanoporous Si [8, 9] require such improved mechanical understanding. For an electro-chemo-mechanical coupled model, one important aspect is

${ }^{*}$ Corresponding Author. E-mail: linder@stanford.edu 

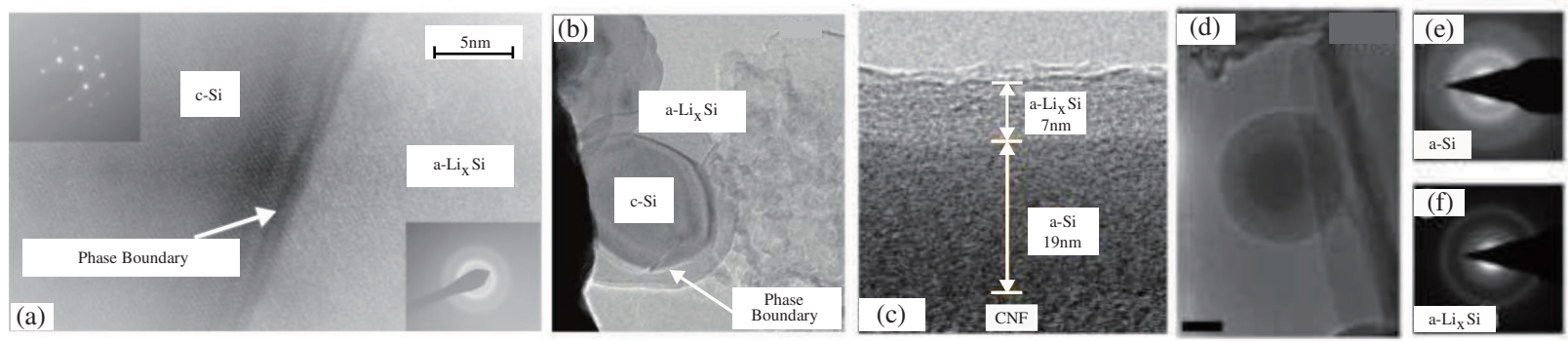

Figure 1: Experimental observation of the two phase lithiation process in c-Si (a,b) and a-Si (c-f). The a-Li $\mathrm{T}_{\mathrm{x}} \mathrm{Si}$ and c-Si (a-Si) form a very sharp phase boundary. (a) Lithiation of c-Si wafer [14], (b) lithiation of c-Si nanoparticle [11], (c) lithiation of a-Si coated on a carbon nanofiber (CNF) [17], (d-f) lithiation of a-Si nanoparticle with the selected area electron diffraction pattern [18].

accurately capturing the $\mathrm{Li}$ distribution in $\mathrm{Si}$ electrodes resulting from the diffusion and reaction process, which is challenging for existing experiments. More importantly, it is necessary to develop a proper model to describe the Li diffusion during the initial lithiation of crystalline $\mathrm{Si}$ (c-Si), amorphous $\mathrm{Si}(\mathrm{a}-\mathrm{Si}$ ) and the subsequent lithiation process, which have different diffusion and reaction behaviors, in a consistent manner. To achieve this, a reaction-controlled diffusion model for the lithiation process of $\mathrm{Si}$ is proposed in this work.

During the past few years, thanks to the in situ transmission electron microscopy electrochemical cells technology [10], many interesting features of diffusion processes of $\mathrm{Li}$ in $\mathrm{Si}$ have been observed. For c-Si, $(i)$ a two-phase diffusion process is reported in [11-13] for the initial lithiation process, as shown in Fig. 1(a,b). As the lithiation proceeds, a layer of amorphous Li-Si alloy $\left(a-\mathrm{Li}_{\mathrm{x}} \mathrm{Si}\right)$, the transparent region in Fig. 1(a,b), is produced. The newly formed $\mathrm{a}-\mathrm{Li}_{\mathrm{x}} \mathrm{Si}$ and the remaining c-Si forms an evident phase boundary (or reaction front). The lithiated a- $\mathrm{Li}_{\mathrm{x}} \mathrm{Si}$ shell may even crystallize into the $\mathrm{Li}_{15} \mathrm{Si}_{4}$ phase during lithiation with the presence of a c-Li core [12]. (ii) The reaction front is observed to have a nanoscale thickness with a high Li concentration gradient [14]. (iii) During the initial lithiation of c-Si nanoparticle electrodes, the reaction front is found to slow down as it progresses towards the core [12]. (iv) The initial lithiation of $\mathrm{c}-\mathrm{Si}$ is anisotropic, resulting in an anisotropic deformation, with a preferred crystal direction $\langle 110\rangle[15,16]$. $(v)$ Fracture of c-Si electrodes during the initial lithiation is size dependent, with a critical diameter of $\sim 150 \mathrm{~nm}$ for nanoparticles $[11,12]$.

For a-Si, (i) recent experiments show a similar two phase diffusion process during initial lithiation [17, 18], as illustrated in Fig. 1(c-f), where a distinct reaction front can be observed between $a-\mathrm{Li}_{\mathrm{x}} \mathrm{Si}$ and a-Si. However, the $\mathrm{Li}$ concentration gradient around the reaction front is not reported in those experiments. (ii) The phase boundary is observed to move at a constant speed [17, 18]. (iii) A two-stage lithiation process is postulated in [17] for a-Si where the $\mathrm{Li}$ concentration first reaches $\mathrm{x} \sim 2.5$ in $\mathrm{a}-\mathrm{Li}_{\mathrm{x}} \mathrm{Si}$, followed by a second lithiation stage when $\mathrm{x} \sim 3.75$. (iv) $\mathrm{A}$ similar size-dependent fracture phenomenon is observed with a critical diameter of $\sim 870 \mathrm{~nm}$ [18]. As for amorphous Si obtained after the delithiation process (named as "post-a-Si" in this work), a one-phase lithiation is observed in experiments [18].

To capture the aforementioned two-phase diffusion mechanism, a purely empirical concentration-dependent diffusion coefficient is commonly used to describe the sharp Li concentration drop in the lithiation of c-Si electrodes $[11,19]$, where the interfacial reaction is ignored and simply replaced by a non-linear diffusion process. A flexible sigmoid function is also used to create Li profiles with either a sharp phase boundary for two-phase lithiation or a gradually varying concentration for one-phase lithiation in [20] without though modeling the diffusion process as done in this work. The Cahn-Hilliard theory [21] for phase separation also captures the phase transformation from $\mathrm{c}-\mathrm{Si}$ to $\mathrm{a}-\mathrm{Li}_{\mathrm{x}} \mathrm{Si}$ in $[22,23]$, where a concentration gradient dependent interfacial contribution in the free energy formulation is used to describe the interfacial region and to control the interfacial size, but the chemical reaction rate is not taken into account. In [24], the concurrent reaction model for the lithiation of c-Si is proposed, where both the stress and reaction rate are accounted for, but the fact that the stress can stall the reaction in the model does not agree with the fact that the reaction front can be extremely slow but never stops [12]. Also, the model is not able to capture concentration gradients at the sharp interface region, which might be a key factor affecting the reaction-limited diffusion process. Similar as in [24], the competition between chemical reaction and species diffusion is treated by a dimensionless parameter in [25], but accounting for large deformations with the consideration of the reaction front velocity, which is assumed to be constant and is thereby not in line with experimental observations for c-Si [12]. 


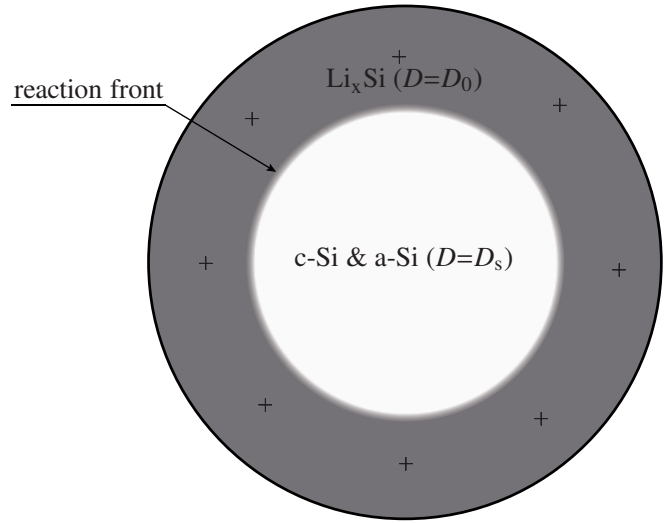

(a) two-phase lithiation for both c-Si and a-Si

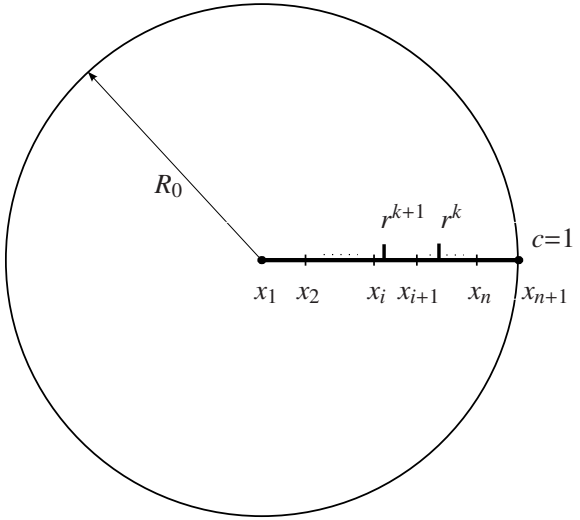

(b) numerical simluation setup

Figure 2: Illustration of the two-phase lithiation mechanism and the numerical simulation setup. (a) The diffusion coefficient $D$ depends on the reaction front location, where $D=D_{0}$ for the obtained a- $\mathrm{Li}_{\mathrm{x}} \mathrm{Si}$ region behind the reaction front and $D=D_{\mathrm{S}}$ for the remaining $\mathrm{Si}$ region ahead of the reaction front. (b) The $1 \mathrm{D}$ simulations in Section 3 represent the lithiation process of Si nanoparticles with an initial radius of $R_{0}$. The finite difference method is used with a second-order central difference approximation of the space derivative and a backward difference approximation of the time derivative. Along the radius, a zero-flux boundary condition is applied at $x_{1}$ and a $c=1$ essential boundary condition is applied at $x_{n+1}$. The radius is discretized into $n$ elements. $r^{k}$ and $r^{k+1}$ represent the reaction front location at time $t=k$ and $t=k+1$, respectively.

For electrodes considered at the nanoscale, the chemical reaction rate rather than the diffusion itself is the limiting factor for the initial lithiation of c-Si and a-Si [11, 12, 24, 25], whereas the diffusion might be the limiting factor for the one-phase diffusion of post-a-Si [18]. To capture the lithiation process for all three types of Si in a uniform way, a one-way coupling model between the reaction and diffusion is proposed in this work. A physical parameter, the bondbreaking energy barrier, is introduced in this new reaction-controlled diffusion model to investigate the similarities and differences between the initial lithiation process of a-Si and c-Si and the lithiation of post-a-Si. The proposed model will take the effect of $\mathrm{Li}$ concentration and hydrostatic pressure at the reaction front on the reaction front velocity into account.

The rest of the paper is organized as follows. Section 2 describes the newly proposed reaction-controlled diffusion model. Several numerical simulations with the consideration of diffusion induced deformation are performed to outline the performance of the proposed model in Section 3. The advantage of the proposed model and its potential applications are discussed in Section 4. Several concluding remarks are given in Section 5.

\section{Reaction-controlled diffusion model}

In diffusion-reaction systems, the concentration of species can be described by a reaction term coupled with the diffusion process through a second order partial differential equation [26]. During the initial lithiation process of Si discussed here, the reaction rate at the phase boundary is a limiting factor of the diffusion process [11-13, 17, 18]. Density functional theory (DFT) simulations show that a Li-concentration threshold value of $c_{0}$ at the reaction front is needed to break the $\mathrm{Si}-\mathrm{Si}$ bond to form dangling bonds [27]. Once the reaction front continues to propagate, Li can diffuse rapidly into $\mathrm{Li}_{\mathrm{x}} \mathrm{Si}$ [25], and the concentration $c$ is governed by the diffusion process in the lithiated region. Therefore, in this case, the instant reaction front velocity under the current lithium concentration profile is more important than the reaction rate at different locations and time instances. Thus, instead of using the full coupling as in [26], the reaction can be decoupled from the diffusion process, where the latter will be used to determine the Li concentration profile. For simplicity, Fick's second law

$$
\frac{\partial c}{\partial t}=D \frac{\partial^{2} c}{\partial x^{2}}
$$

is used, with $D$ as the diffusion coefficient and $c$ as the lithium concentration to determine the Li concentration $c$. The value of $D$ will depend on the location of the reaction front, as shown in Fig. 2(a). For the a-Li $\mathrm{S}_{\mathrm{x}} \mathrm{Si}$ region behind the reaction front, $D=D_{0}$. Although Li could diffuse into c-Si before breaking Si-Si bonds due to a lower diffusion energy 


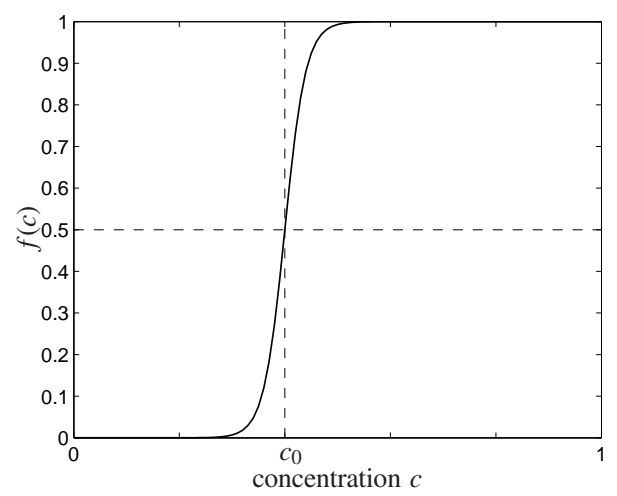

Figure 3: Illustration of the value of $f(c)$ varying with concentration $c$ in (5).

barrier [28], the sharp reaction fronts observed in [14, 17] indicate that very little amount of Li could diffuse across the phase boundary and deposit in the Si core for both c-Si and a-Si. The diffused $\mathrm{Li}$ in unreacted c-Si (or a-Si) might only exist at the dopant level, which is negligible for the results reported here. For post-a-Si, the Si core represents the region to where $\mathrm{Li}$ has not yet diffused. Therefore, we assume $D=D_{\mathrm{s}}=0$ in the unreacted Si core, as shown in Fig. 2a. Alternative diffusion models with a concentration-dependent diffusion coefficient [11] or a pressure-gradient dependent flux term [29] can be used to replace (1).

Having obtained the $\mathrm{Li}$ concentration from (1), the reaction rate between $\mathrm{Li}$ and $\mathrm{Si}$ at the reaction front can be approximated by a solid-state reaction function as

$$
\frac{d \alpha}{d t}=A \exp \left(-\frac{E}{k_{B} T}\right) f(\alpha)
$$

where $\alpha, A, E, k_{B}, T, f(\alpha)$ are the reaction conversion fraction, frequency-related factor, activation energy, Boltzmann constant, absolute temperature, and reaction model, respectively [30]. During the lithiation process of $\mathrm{Si}$, since $\mathrm{Si}$ is the host material, the molar fraction of $\mathrm{Si}$ and $\mathrm{Li}$ is 1 in front of the phase boundary. When the reaction front propagates, $\mathrm{Li}$ will mix with $\mathrm{Si}$ and convert to $\mathrm{Li}_{\mathrm{x}} \mathrm{Si}$. In the newly lithiated region, the $\mathrm{Li}_{\mathrm{x}} \mathrm{Si}$ conversion fraction $\alpha$ in (2) is equivalent to the lithium concentration $c$, and the activation energy $E$ in (2) will be the bond breaking energy needed to break the $\mathrm{Si}-\mathrm{Si}$ covalent bond to form $\mathrm{Li}_{\mathrm{x}} \mathrm{Si}$ alloy under the applied electric potential. Experiments [12], which show a slow-down tendency as the reaction front progresses towards the core during the initial lithiation of c-Si, suggest a stress-dependent reaction front velocity. A more recent experiment conducted with Germanium (Ge), which has a similar crystalline structure to $\mathrm{Si}$, indicates a more evident stress-dependent lithiation process, where a bent Ge nanowire shows a faster lithiation rate under tensile stresses and a slower lithiation speed under compressive stresses. Motivated by those experiments, we propose a hydrostatic pressure dependent reaction rate at the reaction front to replace (2) by

$$
v_{\mathrm{r}}=A \exp \left(-\frac{E_{0}+B p}{k_{B} T}\right) f(c)
$$

where $p$ is the hydrostatic pressure resulting from a diffusion induced deformation and $E_{0}$ is the initial activation energy at a stress-free state already accounting for a possibly applied electric potential [11-13, 17, 18, 31]. The reaction rate (3) will be used to determine the reaction front location. In (3), $B$ is a volume related parameter, which is computed as

$$
B=\frac{\phi_{\mathrm{Si}} V_{\mathrm{Si}}}{2 \cdot 1.60 \cdot 10^{-19}}
$$

where $\phi_{\mathrm{Si}}$ is the $\mathrm{Si}-\mathrm{Si}$ bond fraction in the $\mathrm{Si}$ electrode, $V_{\mathrm{Si}}$ is the atomistic volume of a $\mathrm{Si}$ atom and 2 represents the two $\mathrm{Si}-\mathrm{Si}$ bonds that each $\mathrm{Si}$ atom has. The coefficient $1.60 \cdot 10^{-19}$ in the denominator will ensure that $B p$ represents the bond energy change of a single Si-Si bond with a unit of electronvolt (eV). In (3), compressive stresses (positive hydrostatic pressure) will increase the energy barrier and slow down the reaction rate, and tensile stresses (negative hydrostatic pressure) will decrease the energy barrier and accelerate the reaction rate. 
Table 1: Summary of the parameters used in the simulations performed in Section 3

\begin{tabular}{|l|l||l|l|}
\hline parameter & value & parameter & value \\
\hline \hline$E_{0}(\mathrm{c}-\mathrm{Si})$ & $0.59-0.69(\mathrm{eV})[27,28]$ & $D_{0}$ & $1.0 \cdot 10^{-12}\left(\mathrm{~cm}^{2} / \mathrm{s}\right)[33]$ \\
\hline$E_{0}(\mathrm{a}-\mathrm{Si})$ & $0.51-0.58(\mathrm{eV})$ & $T$ & $300(\mathrm{~K})$ \\
\hline$E_{0}($ post a-Si) & $0.45-0.50(\mathrm{eV})[27,34]$ & $k_{B}$ & $8.6173 \cdot 10^{-5}(\mathrm{eV} / \mathrm{K})$ \\
\hline$c_{0}(\mathrm{c}-\mathrm{Si})$ & $0.10(-)[27]$ & $A$ & $5.0 \cdot 10^{9}\left(\mathrm{~s}^{-1}\right)[35]$ \\
\hline$c_{0}(\mathrm{a}-\mathrm{Si})$ & $0.06(-)$ & $C$ & $50(-)$ \\
\hline$c_{0}($ post a-Si) & $0.001(-)$ & $p_{\max }$ & $1.0-3.5(\mathrm{GPa})$ \\
\hline$\phi_{\mathrm{Si}}(\mathrm{c}-\mathrm{Si})$ & $1.0(-)$ & $\omega$ & $2(-)$ \\
\hline$\phi_{\mathrm{Si}}(\mathrm{a}-\mathrm{Si})$ & $0.8(-)$ & $c_{\max }$ & $8.867 \cdot 10^{4}\left(\mathrm{~mol} / \mathrm{m}^{3}\right)[36]$ \\
\hline$\phi_{\mathrm{Si}}(\mathrm{post} \mathrm{a}-\mathrm{Si})$ & $0.7(-)$ & & \\
\hline
\end{tabular}

A threshold value of $c_{0}$ at the reaction front is needed to break the Si-Si covalent bond. Motivated by [32], where the reaction between near-noble metal and silicon at low temperature arises due to the accumulation of the near-noble metal at the interface, and by DFT calculations in [27], which show a specific threshold of Li concentration above which the Si bond will be broken permanently, a reaction kinetic model $f(c)$ is proposed for $c>0$ as

$$
f(c)=\left(1+\exp \left(C\left(c_{0}-c\right)\right)\right)^{-1}
$$

in a sigmoidal shape, as shown in Fig. (3). Here, $c_{0}$ is the threshold value and $C$ is an empirical parameter to describe the shape of $f(c)$. In this model, $f(c)$ will increase rapidly to a maximum value 1 when the Li concentration $c$ at the reaction front is larger than the threshold value $c_{0}$, and decrease to 0 when $c<c_{0}$. In a given time increment $\Delta t$, the reaction front will propagate a distance $\Delta r=v_{\mathrm{f}} \Delta t$. The reacted Li at the reaction front will break Si-Si bonds and create the path to allow extra Li to diffuse into the Si core. Based on the conservation of species, we approximate the reaction front propagating distance $\Delta r$ as

$$
v_{\mathrm{r}} j d a d t=\Delta r d a c_{0}
$$

with $j$ (number of Li particles/(area-time)) as the Li flux at the reaction front. Here, $j d a d t$ represents the total number of particles that could diffuse across the reaction front. The left hand side of Eq (6) gives the total number of reacted $\mathrm{Li}$ particles. The reacted $\mathrm{Li}$ has to reach the minimum concentration level $c_{0}$, as given at the right hand side of Eq (6), in order to create diffusion paths for Li. The new reaction front location will then be updated as

$$
r^{\text {new }}=r^{\text {old }}-\Delta r
$$

for a cylindrical or spherical geometry [30], where the minus sign indicates that the radius of remaining Si is decreasing due to the reaction.

In our model, the bond-breaking energy barrier $E_{0}$ is the physical quantity which distinguishes different types of $\mathrm{Si}$. It represents the energy barrier to break $\mathrm{Si}$-Si bonds without considering other dynamic processes at the reaction front, such as bond switching or rebonding between Si dangling chains. For the three types of Si discussed in this work, c-Si has the highest bond-breaking energy barrier, due to the well arranged crystalline structure. The energy barrier can be different for different crystalline directions [37], which explains the anisotropic deformation observed in experiments $[15,16]$. The energy barrier in a-Si is expected to be lower than in c-Si due to numerous weak dangling bonds and defects formed during the preparation process [38]. Post-a-Si has the lowest bond-breaking energy barrier, since it contains not only the largest amount of weak dangling bonds, but also voids or trapped Li, as indicated by the $\sim 25 \%$ remaining volume expansion after delithiation [18]. 

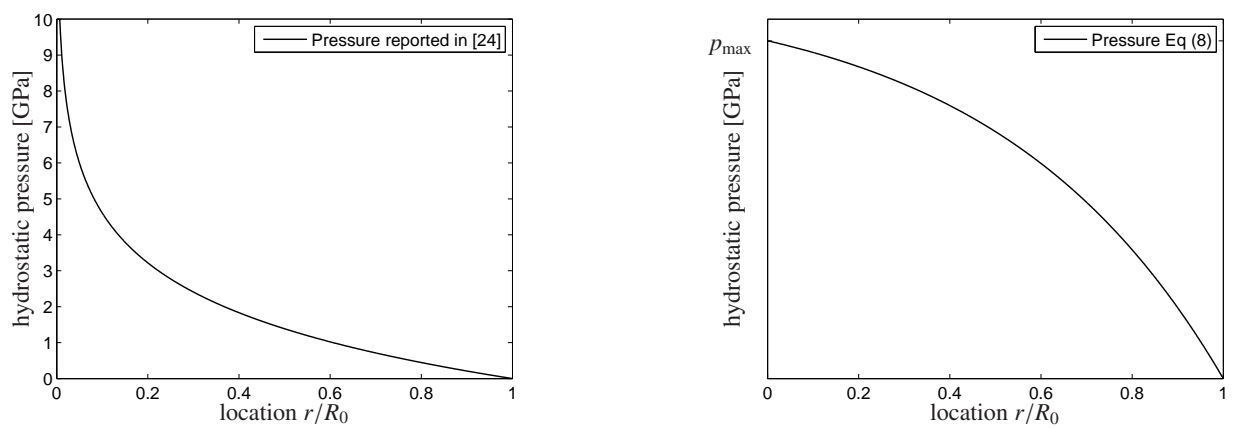

Figure 4: Illustration of the different reaction front pressure values versus radial location used in the simulation, where the left figure represents the pressure reported in [24] and the right one represents the pressure computed from (8).

\section{Numerical simulations}

In this section, several 1D numerical simulations are performed to show the versatility of the proposed model. Those simulations represent the lithiation process of Si nanoparticles with different initial radii of $R_{0}$. We first show the necessity of considering the pressure effects in the proposed model. Then, we carry out a parameter sensitivity study for c-Si, a-Si and post-a-Si, respectively, and compare the numerical simulation with available experimental results. All the simulations are assumed to undergo isotropic deformation without considering different bond-breaking energy barriers for different crystalline directions [28].

In those simulations, the finite difference method is used with a second-order central difference approximation for the space derivative and a backward difference approximation for the time derivative. The chosen parameters used in the simulations are summarized in Table 1. The simulation setup is shown in Fig. 2(b). The radius is discretized into $n=600$ elements and a time step of $1 \cdot 10^{-2} s$ is used. Along the radius, a zero-flux boundary condition is applied at $x_{1}$ and a $c=1$ essential boundary condition is applied at $x_{n+1}$. At time $t=0$, initial conditions in the form of $c=0$ everywhere except at $x_{n+1}$ are prescribed. The initial reaction front $r^{0}$ is located at $x_{n+1}$. At time step $k$, the reaction front is located at $r^{k}$. A zero flux boundary condition is applied for $x<r^{k}$ since $D=0$ for the unlithiated region. The Li concentration profile is computed based on (1), whereas the reaction front velocity at $r^{k}$ is computed based on (3-6). The reaction front location $r^{k+1}$ is then updated based on (7) for the time step $k+1$. The Li flux $j$ at the reaction front in Eq (6) is computed by updating the Li profile through a trial diffusion process without the presence of a reaction front to determine the number of $\mathrm{Li}$ atoms diffusing across the reaction front per second. For post-a-Si, the predicted reaction velocity might be larger than the diffusion speed due to smaller chosen $E_{0}$. To ensure that the reaction can only happen when $\mathrm{Li}$ is present, a cutoff value of $\mathrm{Li}$ concentration $c_{\text {cutoff }}=c_{0} / 10$ is used to determine the reaction front location. The simulation repeats for the new time step $k+1$.

To account for the diffusion induced deformation, we consider a linear relationship between the concentration and the deformation $\Delta R / R=\Omega \Delta c$ as in [19, 39]. An expansion coefficient $\Omega=0.6$ as in [19] is used to obtain the $\sim 300 \%$ volume expansion at fully lithiated state. In the simulation procedure, the averaged concentration level in each discretized segment is computed first. Then, the corresponding segment undergoes a concentration level driven deformation. The concentration level at the next time step will be computed based on the deformed geometry. For Fig. 5 to Fig. 15, the concentration profile versus radial location curves are plotted for the swelled geometry. The makers on the upper right corner of each figure indicate the new radius of the swelled Si electrodes at the corresponding time step.

\subsection{Pressure effects on the lithiation process}

In this subsection, we investigate the effect of hydrostatic pressure values at the reaction front on the lithiation process, which is taken into account in (3). We run simulations with three bond-breaking energy barriers $E_{0}$ corresponding to c-Si, a-Si and post-a-Si. It is shown that by considering a proper value for the hydrostatic pressure, the two-phase lithiation process for c-Si, a-S and the one-phase lithiation process for post-a-Si is captured by the proposed model.

For a nanoparticle undergoing ideal plastic deformation, the reaction front pressure can be calculated based on $p=2 \sigma_{Y} \log \left(r / R_{0}\right)$ [24]. As in [24], $\sigma_{Y}=1 \mathrm{GPa}$ is chosen and the pressure versus radial location is plotted on the left of 


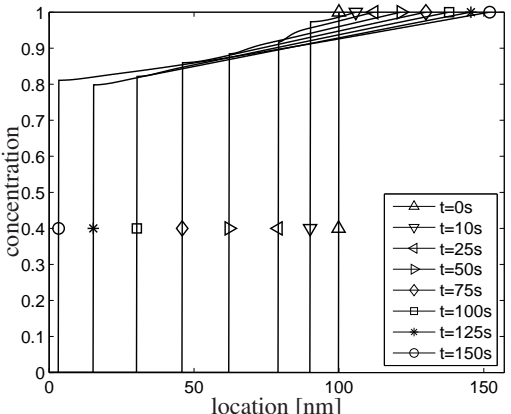

(a) concentraton - without pressure

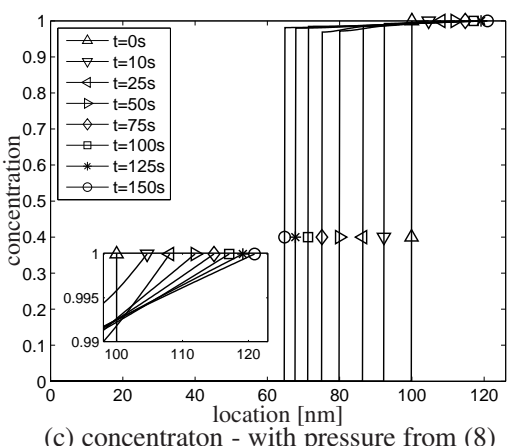

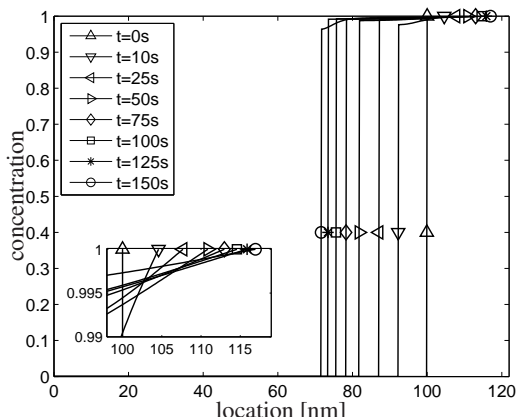

(b) concentraton - with pressure from [24]

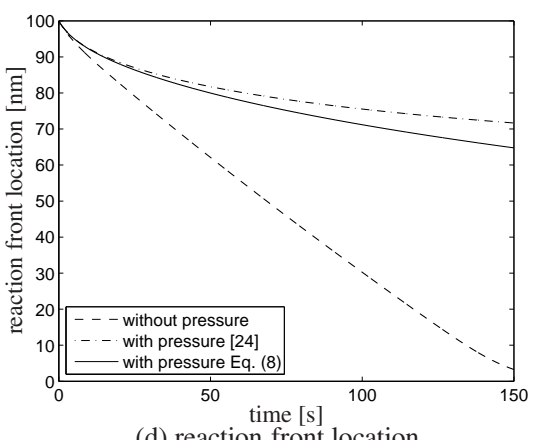

(d) reaction front location

Figure 5: c-Si simulation - Illustration of the concentration profile versus radial location at different time steps for $E_{0}=0.69 \mathrm{eV}(\mathrm{a}-\mathrm{c}$ ), and the reaction front location versus time (d) corresponding to (a-c). (a-c) An evident reaction front is observed for each simulation. The Li concentration profile is high in the lithiated region. (d) The reaction front velocity is constant when the pressure is neglected and slows down when the pressure is included.

Fig. 4. Since the plastic deformation is assumed to be unaffected by the superimposed hydrostatic stress in [24], the hydrostatic pressure approaches an infinite value at the core.

Motivated by [40], where the yield stress is related to the hydrostatic pressure and a finite yield stress should correspond to a finite hydrostatic pressure, a location dependent empirical reaction front pressure distribution with a saturated value $p_{\max }$, as an alternative to the one reported in [24], is proposed as

$$
p=p_{\max }\left(1-\exp \left(-\omega\left(R_{0}-r\right) / R_{0}\right)\right)
$$

where $\omega$ is a scalar parameter approximating the shape of the reaction front pressure distribution during the diffusion induced plastic deformation. Since currently no fully chemo-mechanical coupled formulation accounting for DruckerPrager plasticity given in the literature provides pressure distribution at the reaction front, we perform a quantitative study based on the pressure distribution given in [24] and the empirical reaction front pressure distribution in (8).

To do so, the two pressure distributions illustrated in Fig. 4 are now inserted into (3) to study the pressure effect on the lithiation process for the three different values of the bond-breaking energy barrier $E_{0}$ with $p_{\max }=1.5 \mathrm{GPa}$. For c-Si, a nanoparticle with a radius $R_{0}=100 \mathrm{~nm}$ is studied with $E_{0}=0.69 \mathrm{eV}$ and $c_{0}=0.1$ [27]. For post-a-Si, the one-phase lithiation process indicates a diffusion-controlled lithiation process. The $\mathrm{Si}$-Si bonds break at the same speed as the diffusion process. Therefore, a value of $E_{0}=0.50 \mathrm{eV}$, corresponding to the diffusion energy barrier of a-Si [34], is used to study the lithiation process of a post-a-Si nanoparticle with a radius $R_{0}=250 \mathrm{~nm}$. A very small but non-zero value of $c_{0}(=0.001)$ is used for the purpose of determining the reaction front location in the numerical simulation. For a-Si, a nanoparticle with a radius $R_{0}=250 \mathrm{~nm}$ is studied with $E_{0}=0.58 \mathrm{eV}$ and $c_{0}=0.06$. Since no universal agreement about the bond-breaking energy barrier exists for c-Si, a-Si and post-a-Si, we can only estimate the bond-breaking energy barrier range based on DFT calculations [27, 28, 34], as given in Table 1, and perform parameter studies in later subsections.

The Li concentration profile versus radial location at different time steps for c-Si is shown in Fig. 5(a-c) with and without the consideration of the pressure effects. For the lithiation process of c-Si, a sharp reaction front can be observed in all three simulations in Fig. 5(a-c). A high Li concentration is obtained in the lithiated region, matching 


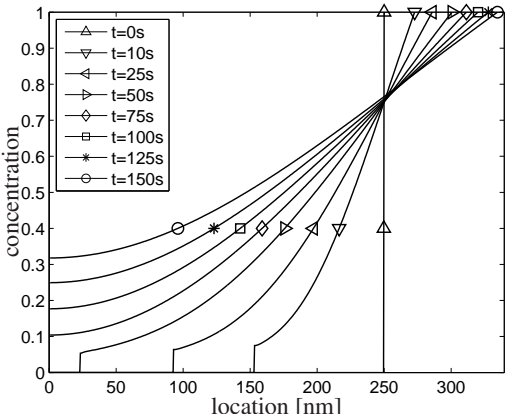

(a) concentraton - without pressure

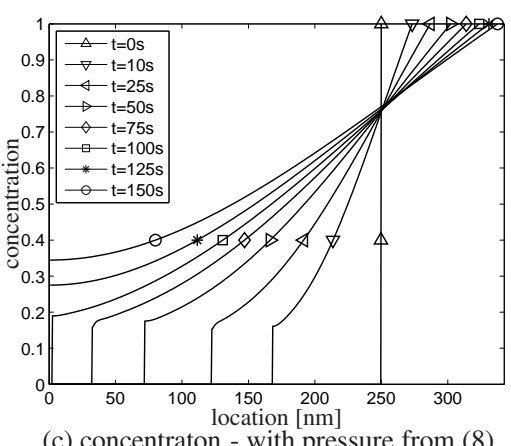

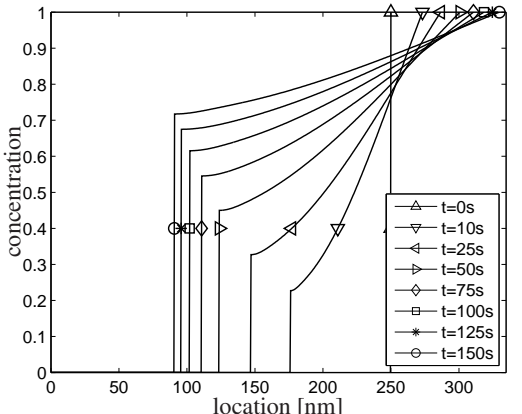

(b) concentraton - with pressure from [24]

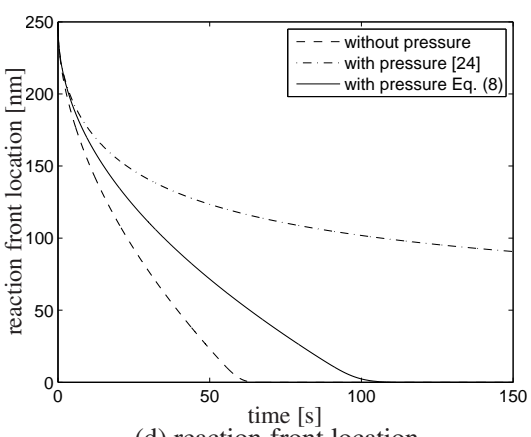

(d) reaction front location

Figure 6: a-Si simulation - Illustration of the concentration profile versus radial location at different time steps for $E_{0}=0.58 \mathrm{eV}$ (a-c), and the reaction front location versus time (d) corresponding to (a-c). (a-c) An evident reaction front is observed for each simulation. (d) The reaction front velocity slows down significantly due to the pressure [24].

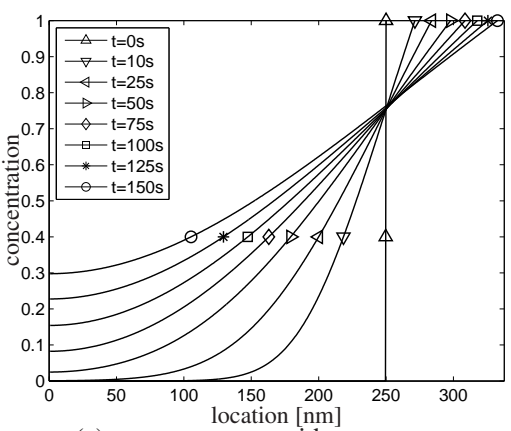

(a) concentraton - without pressure

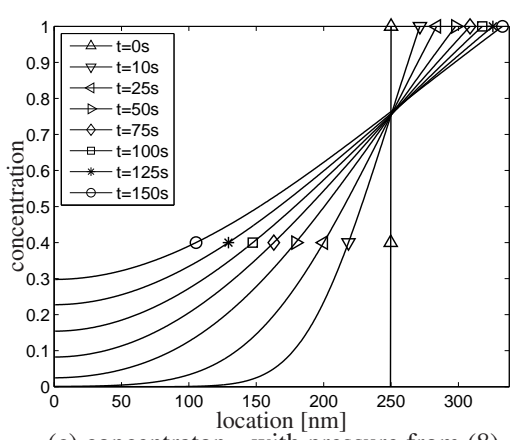

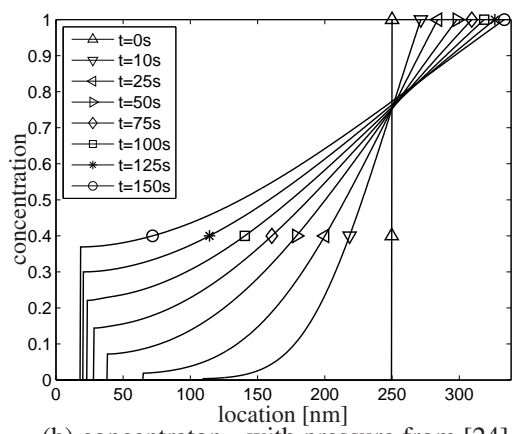

(b) concentraton - with pressure from [24]

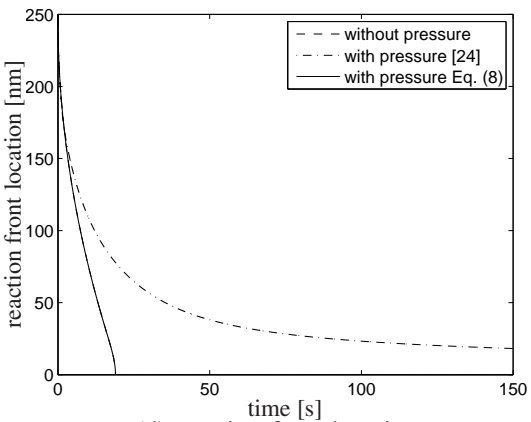

(d) reaction front location

Figure 7: post-a-Si simulation - Illustration of the concentration profile versus radial location at different time steps for $E_{0}=0.50 \mathrm{eV}$ (a-c), and the reaction front location versus time (d) corresponding to $(\mathrm{a}-\mathrm{c})$. (a,c) A one-phase lithiation process is observed for both simulations. (b) the high pressure effect alternates the lithiation process from one-phase to two-phase. (d) The reaction front velocity moves as fast as the diffusion process for (a) and (c) and slows down for (b). 


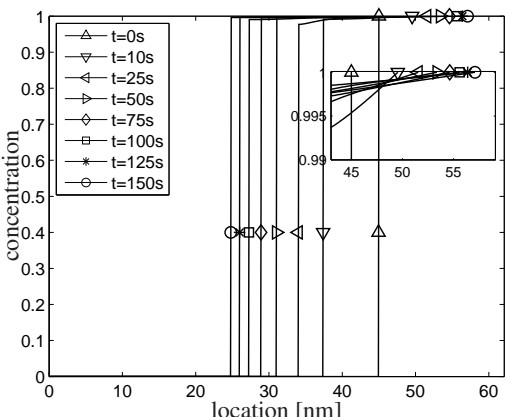

(a) concentration - $E_{0}=0.67 \mathrm{eV}$

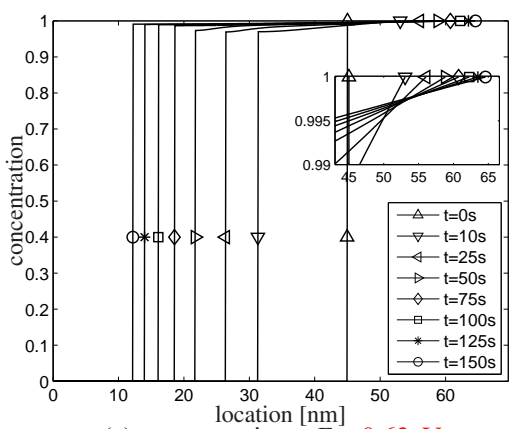

(c) concentration $-E_{0}=0.63 \mathrm{eV}$

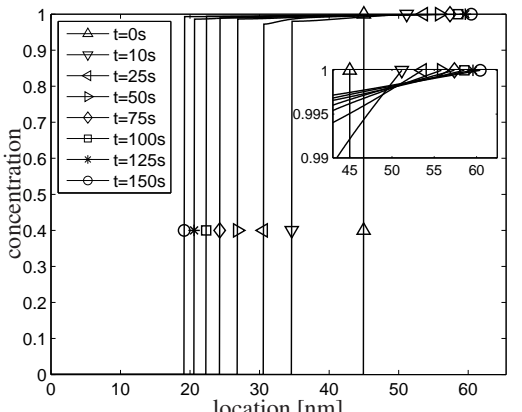

(b) concentration $-E_{0}=0.65 \mathrm{eV}$

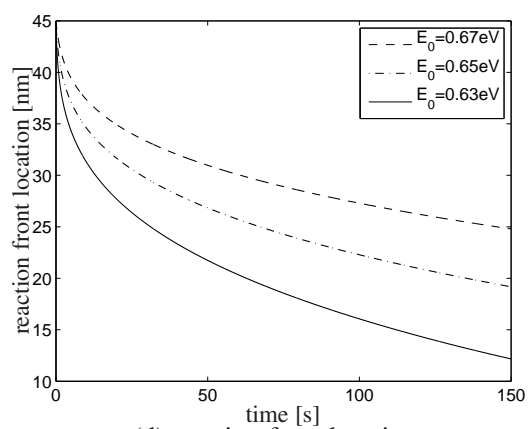

(d) reaction front location

Figure 8: c-Si simulation - Illustration of the concentration profile versus radial location at different time steps for different $E_{0}$ with $p_{\text {max }}=2.5 \mathrm{GPa}$. The bond-breaking energy barrier significantly affects the lithiation speed. For smaller value of $E_{0}$, more silicon is lithiated.

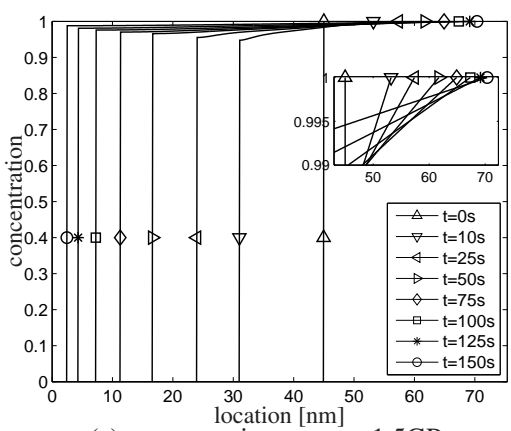

(a) concentration $-p_{\max }=1.5 \mathrm{GPa}$

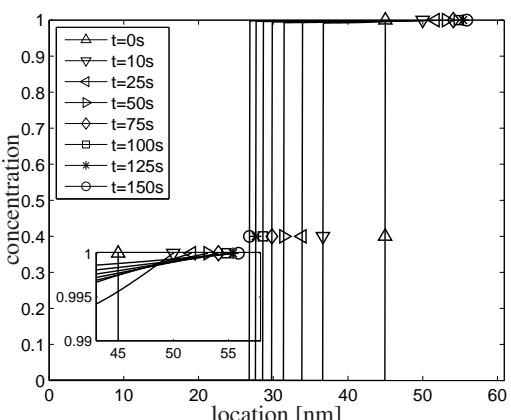

(c) concentration $-p_{\max }=3.5 \mathrm{GPa}$

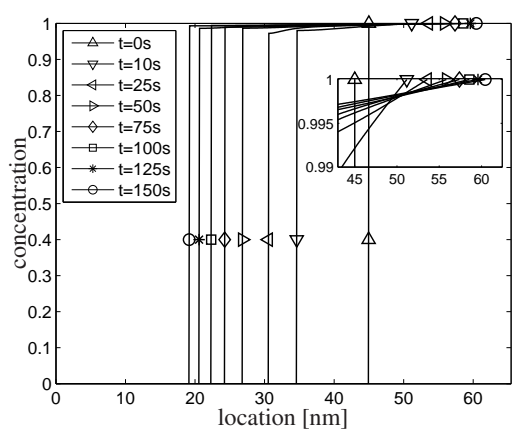

(b) concentration $-p_{\max }=2.5 \mathrm{GPa}$

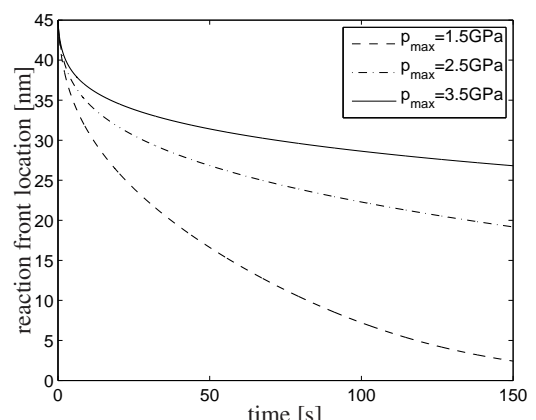

(d) reaction front location

Figure 9: c-Si simulation - Illustration of the concentration profile versus radial location at different time steps for different $p_{\text {max }}$ with the same $E_{0}=0.65 \mathrm{eV}$. Like the bond-breaking energy barrier, the reaction front pressure significantly affects the lithiation speed. For smaller value of $p_{\text {max }}$, more silicon is lithiated. 


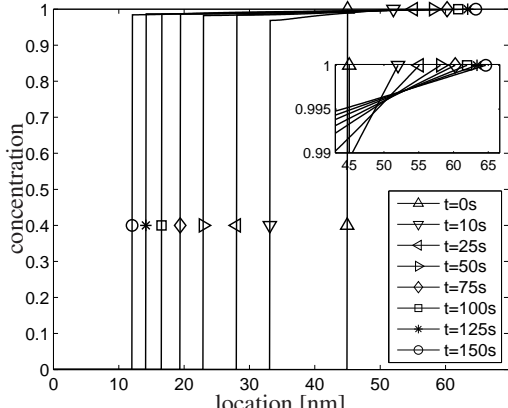

(a) $R_{0}=45 \mathrm{~nm}$

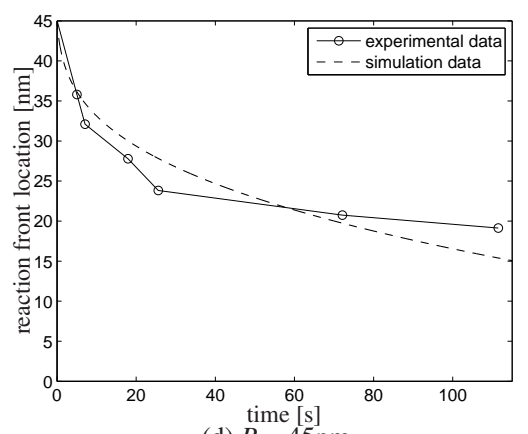

(d) $R_{0}=45 \mathrm{~nm}$

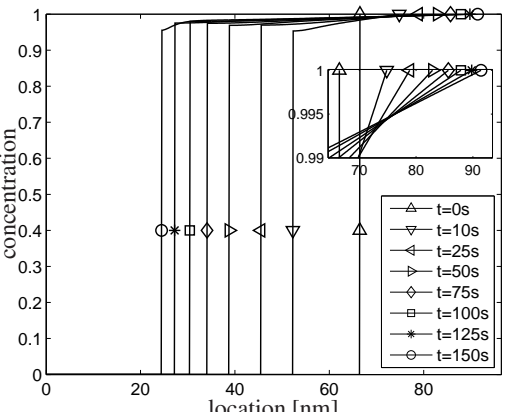

(b) $R_{0}=66.5 \mathrm{~nm}$

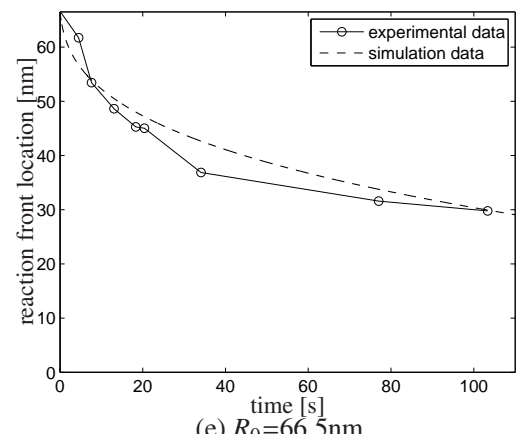

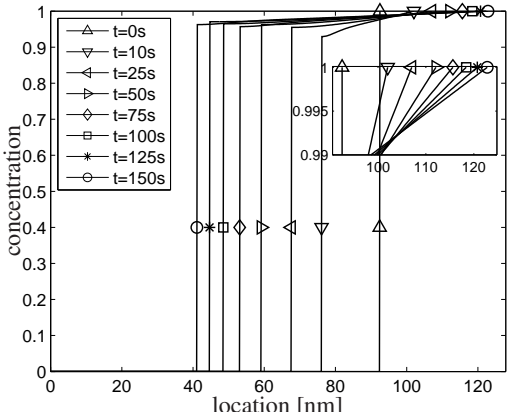

(c) $R_{0}=92.5 \mathrm{~nm}$

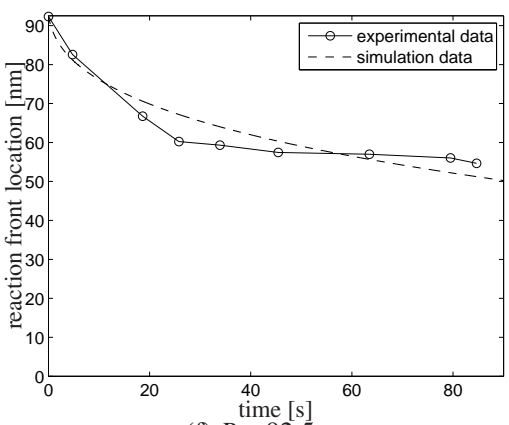

(f) $R_{0}=92.5 \mathrm{~nm}$

Figure 10: c-Si simulation - Illustration of the concentration profile versus radial location at different time steps for $E_{0}=0.65 \mathrm{eV}$ and $p_{\max }=2.0 \mathrm{GPa}$ (a-c), and the reaction front location versus time (d-f) with the comparison of the experimental data for three different initial radii. (a-c) An evident reaction front is observed. In the lithiated region, a high Li concentration level is obtained from the simulation. (d-f) The reaction front location curves match the experimental observation and the gradient of those curves indicate a slow-down tendency for the reaction front velocity.

the experimental observation [12]. The reaction front location versus time is plotted in Fig. 5(d). For the simulation with pressure in [24] and (8), the reaction front velocity shows a slow-down behavior, matching the experimental observation where the reaction front velocity becomes smaller as the reaction front approaches the core. This is not the case when neglecting the pressure effect for which an almost constant reaction velocity is obtained, contradicting experimental results [12].

For a-Si, the Li concentration profile versus radial location at different time steps is shown in Fig. 6(a-c) with and without the consideration of the pressure effect. The simulations in Fig. 6(a-c) recover the two-phase lithiation process as observed in experiments [18]. The pressure from [24] causes the lithiation process to stop, as shown in Fig. 6(b), contradicting experimental observations [18], due to an infinite pressure in [24] at the core of nanoparticles.

For post-a-Si, the Li concentration profile versus radial location at different time steps is shown in Fig. 7(a-c) with and without the consideration of the pressure effect. The simulations in Fig. 7(a) and (c) are reasonable and recover the one-phase lithiation process as observed in experiments [18]. The pressure from [24] plays an exaggerated effect on the reaction rate and alternates the lithiation process from one-phase to two-phase, as shown in Fig. 7(b), due to the infinite pressure value at the core.

The three simulations in Fig. 5 indicate the importance of the consideration of the reaction front pressure effects in the proposed model. The drastic differences in the two pressure formulations used in Fig. 6(b,c) and 7(b,c) further outline the importance of the hydrostatic pressure effect. In the remaining part of this section, we will only consider the simulations with the pressure effect in (8) but not the pressure formulation with an infinite value at the core of nanoparticles in [24]. In the future, a chemo-mechanical coupled model will be used to study the diffusion induced deformation in order to obtain a more realistic pressure value to be inserted into (3).

\subsection{Lithiation of crystalline silicon nanoparticles}

Values for the bond-breaking energy barrier can only be obtained from DFT calculations. To illustrate the impact of the bond-breaking energy barrier and the pressure distribution on the lithiation process, we carry out a parameter sensitivity study for c-Si with $E_{0}$ varying from $0.59 \mathrm{eV}$ to $0.69 \mathrm{eV}$ and $p_{\max }$ varying from $1.0 \mathrm{GPa}$ to $3.5 \mathrm{GPa}$. Here, we 


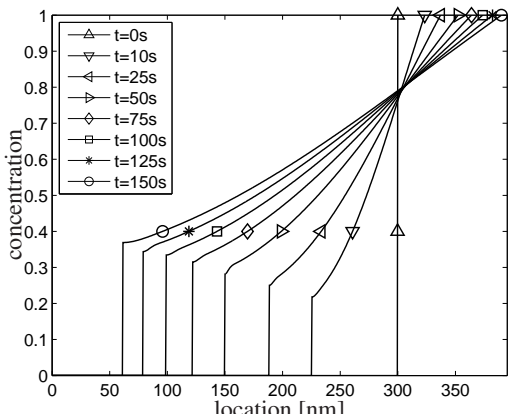

(a) concentration - $E_{0}=0.58 \mathrm{eV}$

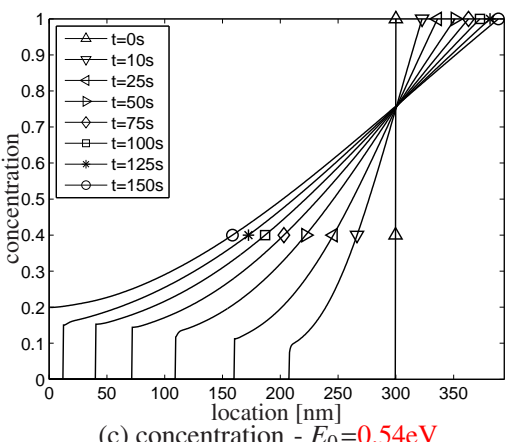

(c) concentration - $E_{0}=0.54 \mathrm{eV}$

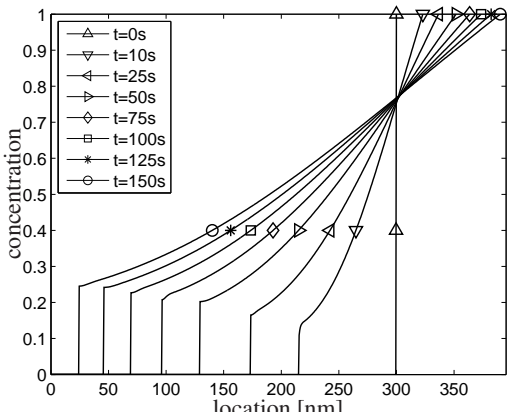

(b) concentration - $E_{0}=0.56 \mathrm{eV}$

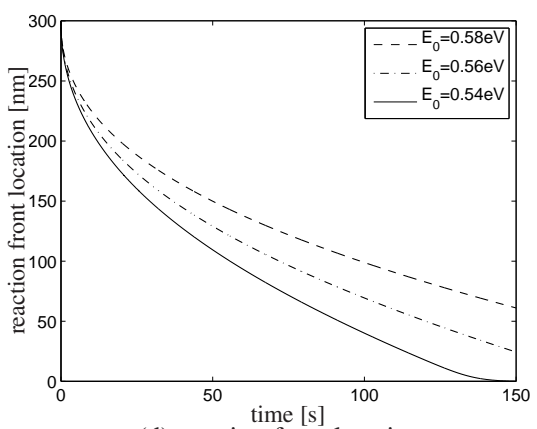

(d) reaction front location

Figure 11: a-Si simulation - Illustration of the concentration profile versus radial location at different time steps for different $E_{0}$ with $p_{\text {max }}=2.5 \mathrm{GPa}$ and $R_{0}=300 \mathrm{~nm}$. The bond-breaking energy barrier significantly affects the concentration profiles and the lithiation speed. The Li concentration level is higher in the lithiated region for larger $E_{0}$, but is much lower than the concentration level for c-Si in Fig. 8. Similar as for c-Si, the lithiation speed is slower for larger $E_{0}$ as shown in (d).

keep one parameter constant and vary the other one. Fig. 8 shows the concentration profile versus radial location at different time steps and the reaction front location versus time for a maximum pressure $p_{\max }$ fixed at $2.5 \mathrm{GPa}$ while varying $E_{0}$ from $0.69 \mathrm{eV}$ to $0.59 \mathrm{eV}$, with $R_{0}=45 \mathrm{~nm}$. Fig. 8 indicates that the bond-breaking energy barrier significantly affects the lithiation speed. For smaller value of $E_{0}$, more silicon is lithiated. The concentration profile and reaction front location curves for $E_{0}=0.65 \mathrm{eV}$ and $p_{\max }$ varying from $1.0 \mathrm{GPa}$ to $3.5 \mathrm{GPa}$ are shown in Fig. 9. It can be observed that the pressure has an impact on lithiation speed as well. More silicon is lithiated for a smaller $p_{\max }$.

The numerical results are compared with the available experimental data for nanoparticles in [12]. For one pair of the parameters, $E_{0}=0.65 \mathrm{eV}$ and $p_{\max }=2.0 \mathrm{GPa}$, we show the concentration profile versus radial location in Fig. 10(ac), and the reaction front location versus time with comparison to experimental data in Fig. 10(d-f), for three different initial radii. In the $\mathrm{Li}$ concentration profile plot, the sharp reaction front is recovered by the simulation. A high Li concentration is obtained in the lithiated region with $c \approx 1.0$, as shown in Fig. 10(a-c), which matches the experimental observation that $\mathrm{Li}_{3.75} \mathrm{Si}$ is present with a remaining c-Si core in the initial lithiation process of c-Si nanoparticles [12]. The reaction front locations, as shown in Fig. 10(d-f), matches the experimental observation in [12].

\subsection{Lithiation of amorphous silicon nanoparticles}

Similar to c-Si, we perform the parameter sensitivity study for a-Si with $E_{0}$ varying from $0.51 \mathrm{eV}$ to $0.58 \mathrm{eV}$ and $p_{\max }$ varying from 1.0GPa to $3.5 \mathrm{GPa}$. Fig. 11 shows the concentration profile versus radial location at different time steps and the reaction front location versus time for a maximum pressure $p_{\max }=2.5 \mathrm{GPa}$ and a varying $E_{0}$, changing from $0.58 \mathrm{eV}$ to $0.51 \mathrm{eV}$, with $R_{0}=300 \mathrm{~nm}$. Fig. 11 indicates that the bond-breaking energy barrier significantly affects the concentration profiles and the lithiation speed. The Li concentration level is higher in the lithiated region for larger $E_{0}$, but is much lower than the concentration level for c-Si in Fig. 8. Similar to c-Si, the lithiation speed is slower for larger $E_{0}$ as shown in Fig. 11(d). The concentration profile and reaction front location curves for $E_{0}=0.56 \mathrm{eV}$ and $p_{\max }$ varying from 1.0GPa to $3.5 \mathrm{GPa}$ are shown in Fig. 12. It can be observed that the pressure has an impact on the concentration profiles and the lithiation speed. The Li concentration level is higher in the lithiated region for higher $p_{\max }$. Similar as for c-Si, the lithiation speed is slower for larger $p_{\max }$. 


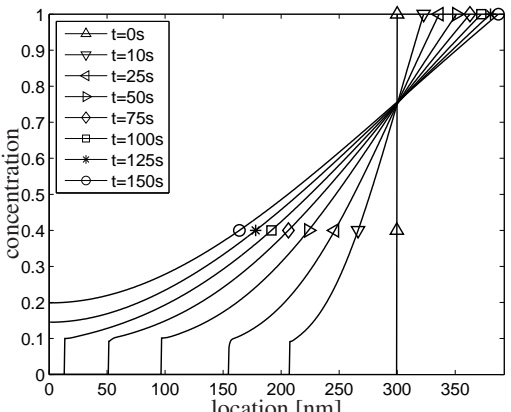

(a) concentration $-p_{\max }=1.5 \mathrm{GPa}$

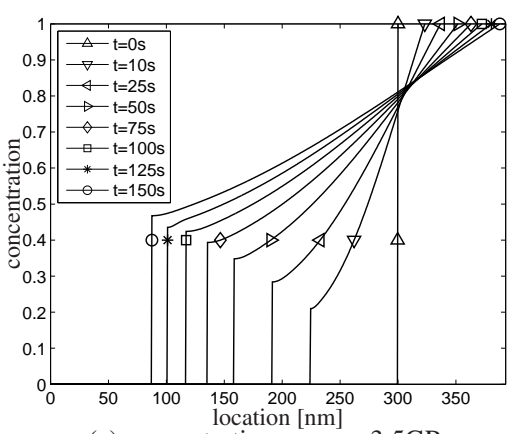

(c) concentration $-p_{\max }=3.5 \mathrm{GPa}$

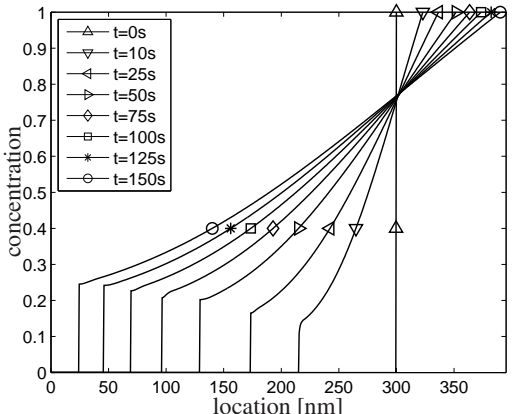

(b) concentration $-p_{\max }=2.5 \mathrm{GPa}$

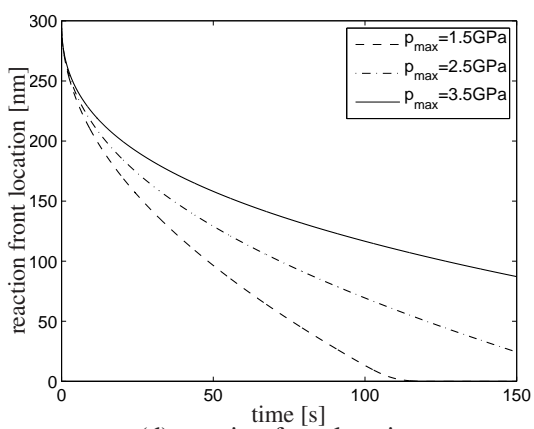

(d) reaction front location

Figure 12: a-Si simulation - Illustration of the concentration profile versus radial location at different time steps for different $p_{\text {max }}$ with $E_{0}=0.56 \mathrm{eV}$ and $R_{0}=300 \mathrm{~nm}$. The reaction front pressure significantly affects the concentration profiles and the lithiation speed. The Li concentration level is higher in the lithiated region for higher pressure. Similar as for c-Si, the lithiation speed is slower for larger $p_{\max }$.
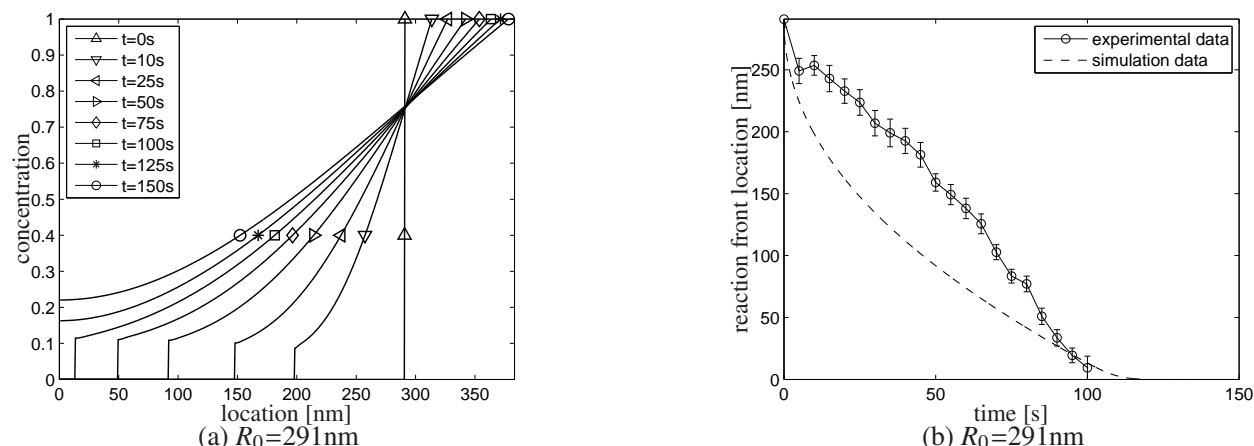

Figure 13: a-Si simulation - Illustration of the concentration profile versus radial location at different time steps for $E_{0}=0.55 \mathrm{eV}$ (left), and the reaction front location versus time (right) with comparison of the experimental data.

The numerical results are compared with the experimental data in the supporting file of [18]. For one pair of the parameters $E_{0}=0.55 \mathrm{eV}$ and $p_{\max }=1.9 \mathrm{GPa}$, we show the concentration profile versus radial location in Fig. 13(a) and the reaction front location versus time with comparison to experimental data in Fig. 13(b) for $R_{0}=291 \mathrm{~nm}$. The reaction front location from the simulation is comparable to the experimental observation as shown in Fig. 13(b).

\subsection{Lithiation of post amorphous silicon nanoparticles}

For post-a-Si nanoparticle with $R_{0}=300 \mathrm{~nm}$, the parameter sensitivity study is carried out with $E_{0}$ varying from $0.45 \mathrm{eV}$ to $0.50 \mathrm{eV}$ and $p_{\max }$ varying from $1.0 \mathrm{GPa}$ to $3.5 \mathrm{GPa}$. Similar to c-Si and a-Si, we first run simulations with different $E_{0}$, while keeping $p_{\max }=2.5 \mathrm{GPa}$ constant. Then, we investigate the pressure effect with $E_{0}=0.48 \mathrm{eV}$ and varying $p_{\max }$. The concentration profile versus radial location at different time steps and the reaction front location versus time for those two sets of simulations are plotted in Fig. 14 and 15, respectively. From Fig. 14, it can be observed the one phase lithiation process is recovered for all the different bond-breaking energy barriers under the given pressure effect. The lithiation process proceeds as fast as the diffusion process. The different bond-breaking 


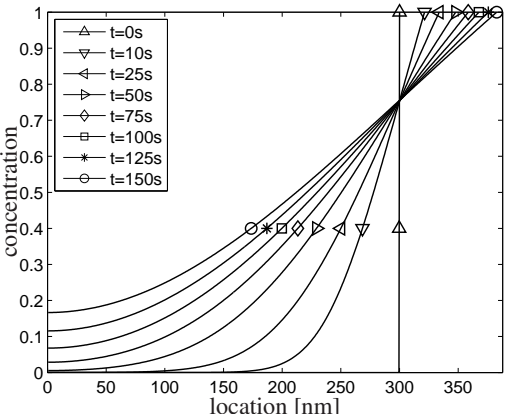

(a) concentration - $E_{0}=0.50 \mathrm{eV}$

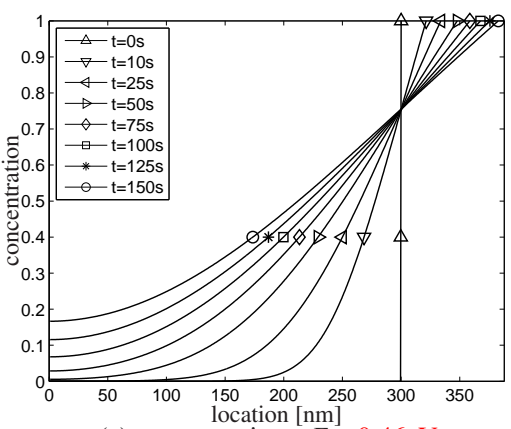

(c) concentration - $E_{0}=0.46 \mathrm{eV}$

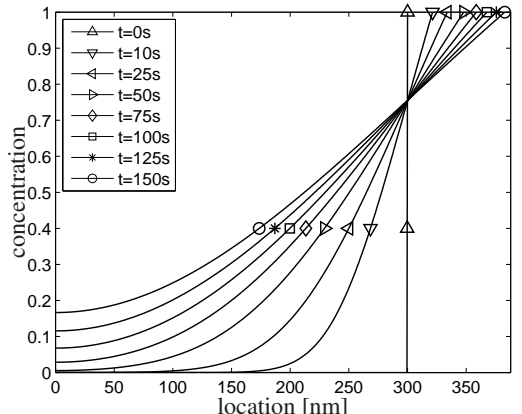

(b) concentration $-E_{0}=0.48 \mathrm{eV}$

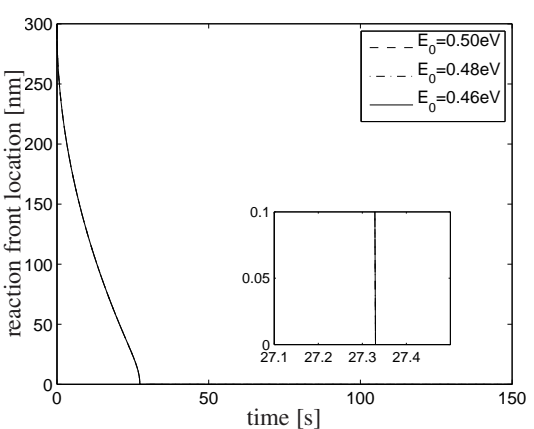

(d) reaction front location

Figure 14: post-a-Si simulation - Illustration of the concentration profile versus radial location at different time steps for different $E_{0}$ with $p_{\max }=2.5 \mathrm{GPa}$ for $R_{0}=300 \mathrm{~nm}$. Since the bond-breaking energy barriers used here are very small, the one phase lithiation process is recovered for all the different energy barriers under the given pressure effect. The lithiation process proceeds as fast as the diffusion process.

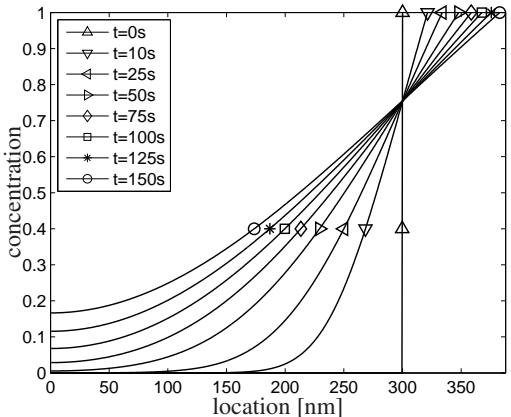

(a) concentration $-p_{\max }=1.5 \mathrm{GPa}$

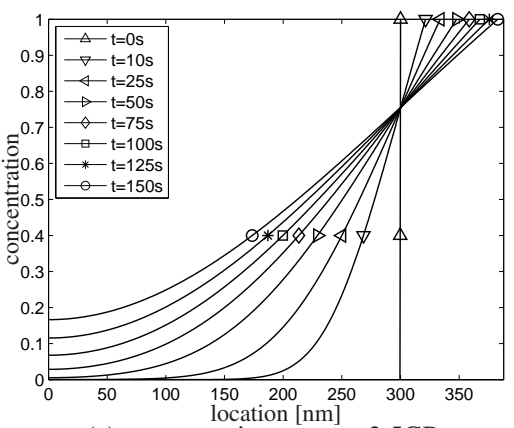

(c) concentration $-p_{\max }=3.5 \mathrm{GPa}$

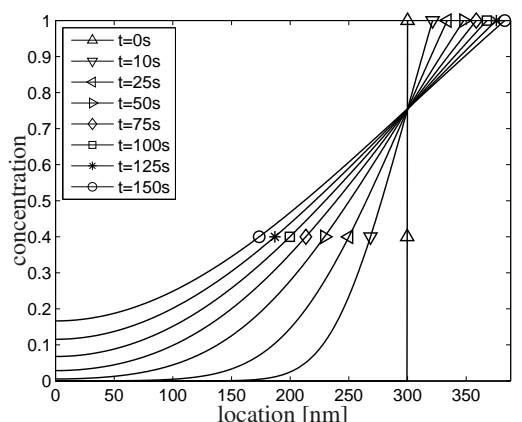

(b) concentration $-p_{\max }=2.5 \mathrm{GPa}$

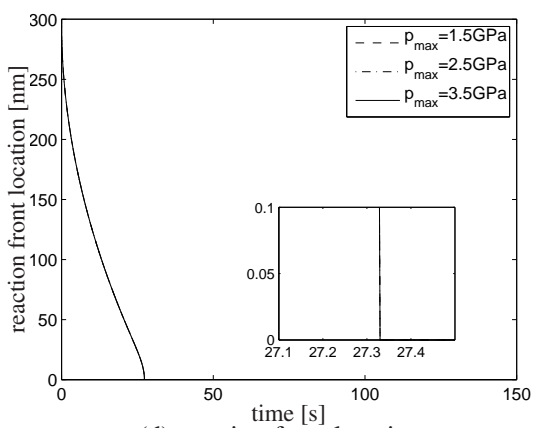

(d) reaction front location

Figure 15: post-a-Si simulation - Illustration of the concentration profile versus radial location at different time steps for different $p_{\text {max }}$ with the same $E_{0}=0.48 \mathrm{eV}$ for $R_{0}=300 \mathrm{~nm}$. The reaction front hydrostatic pressure has less effect on the concentration profiles and the lithiation speed, when compared with Fig. 9 and 12 . 
energy barriers $E_{0}$ show only a miner effect on the lithiation process. Fig. 15 shows the influence of the hydrostatic pressure on the lithiation process. Like the bond-breaking energy barriers, also the hydrostatic pressure does not change the lithiation process significantly. However, if the hydrostatic pressure at the reaction front in the Si core approaches an infinite value as shown in Fig. 7(b), the concentration profile from the numerical simulation might be misleading. But since also the distribution in Equation (8) is of empirical nature, we are currently working on a fully chemo-mechanical coupled model accounting for a Drucker-Prager type plasticity to automatically provide us with the missing information of the real pressure distribution at the reaction front.

\section{Discussion}

The simulations carried out in Section 3 outline the performance of the proposed model and support the consideration of the reaction and diffusion separately. Also, the numerical simulation support the account of the pressure effect on the reaction rate. By varying certain physical material parameters, such as the bond-breaking energy barrier and the initial threshold of Li concentration, which can be obtained from DFT calculations, the proposed model is able to describe the lithiation process of all three types of Si discussed here.

The proposed model can be easily implemented within existing diffusion models and thereby improve the computational efficiency without introducing additional degrees of freedom. Since the chemical reaction is coupled with the $\mathrm{Li}$ concentration in one way, the reaction rate is determined based on the computed Li concentration profile on a local level. Consequently, the reaction front location is determined locally, differing from [25], where the reaction front location is introduced as one additional degree of freedom. Because of the automatically generated two phase diffusion process with a high Li concentration drop at the reaction front, the usage of the Cahn-Hilliard theory to capture the phase transformation in the continuum model [22,23], which would require the introduction of one additional degree of freedom when employing the finite element method to solve the 4th order partial differential equation with a piecewise linear approximation of the Li concentration, can be avoided.

By considering the reaction and diffusion process separately, rather than considering the reaction as a non-linear diffusion process, the proposed model is capable of providing additional insight to understand the competition between diffusion and reaction processes during (de)lithiation. It also suggests different ways to mitigate high concentration gradients at c-Si. If particular non-damage technologies like, X-ray, heating, applied electrical field, magnetic field, could be used to agitate the $\mathrm{c}-\mathrm{Si}$, the reaction process might be increased and the stress state will be altered, leading to an improvement of the performance of c-Si. Also, the critical size of a-Si might be increased by doping technologies which decrease the energy barrier and mitigate the concentration at the interface region.

The proposed model is capable to study the lithiation process involving multiple phases. A two-stage lithiation process is postulated in [17] for a-Si, based on computing the average Li concentration in the lithiated region, where the $\mathrm{Li}$ concentration first reaches $\mathrm{x} \sim 2.5$ in $\mathrm{a}-\mathrm{Li}_{\mathrm{x}} \mathrm{Si}$, followed by a second lithiation stage when $\mathrm{x} \sim 3.75$. If it is true that there exists a second phase of lithiation for a-Si, in addition to the initial bond-breaking energy barrier needed to break the covalent bonds to create the diffusion paths, a second energy barrier and an additional reaction front will be needed to capture the second lithiation stage. However, the simulation of such two-stage lithiation with the proposed model is not attempted in this work, due to the lack of experimental information for the second stage lithiation. As shown in the first simulation for $E_{0}=0.58 \mathrm{eV}$, the concentration gradient is small at the interfacial region. The obtained $\mathrm{x} \sim 2.5$ in the experiment [17] might be caused by incomplete diffusion, since there is no evidence that shows that the lithiated $\mathrm{a}-\mathrm{Li}_{\mathrm{x}} \mathrm{Si}$ has a homogeneous lithium distribution.

For other materials like Germanium, which involves different phases during the lithiation and delithiation process, such as $\mathrm{Li}_{9} \mathrm{Ge}_{4}, \mathrm{Li}_{\mathrm{x}} \mathrm{Ge}$ and $\mathrm{Li}_{15} \mathrm{Ge}_{4}$ [31], this model can be very useful. If the multiple phases are caused by different bond-breaking energy barriers at different applied electric potential levels, with additional information about the physical quantities such as different bond-breaking energy barriers, diffusion coefficients, and different concentration threshold levels, the proposed model will be capable to capture very complicated (de)lithiation processes of Germanium by introducing multiple energy barriers and reaction fronts.

\section{Conclusion and outlook}

In this work, a reaction-controlled diffusion model is proposed to universally describe the lithiation process for three types of $\mathrm{Si}$ based on the bond-breaking energy barrier $E_{0}$ as the key physical material parameter. In addition, 
the numerical simulations demonstrate the importance of accounting for the hydrostatic pressure at the reaction front on the reaction rate. By treating diffusion and reaction separately, this model might suggest different ways to advance the performance of $\mathrm{Si}$ by either improving the chemical reaction speed or diffusion property between $\mathrm{Li}$ and $\mathrm{Si}$. Our model also has the potential to explain the complicated diffusion induced phase transformation in other materials, such as Germanium. The proposed model is very easy to implement into existing finite element codes, so that in the future, we will be in the position to study the diffusion induced deformation and fracture of Si anode material in a full electro-chemo-mechanical coupled way by incorporating this model to our computational fracture framework [41-51]. We will perform DFT calculations $[52,53]$ to calibrate the physical parameters used in the proposed model.

\section{Acknowledgment}

Financial support was provided by the Samsung Global Research Partnership and the Professor James M. Gere Graduate Fellowship from Stanford University. This support is gratefully acknowledged.

\section{References}

[1] M. T. McDowell, S. W. Lee, W. D. Nix, Y. Cui, 25th anniversary article: Understanding the lithiation of silicon and other alloying anodes for lithium-ion batteries, Advanced Materials 25 (2013) 4966-85.

[2] U. Kasavajjula, C. Wang, A. J. Appleby, Nano- and bulk-silicon-based insertion anodes for lithium-ion secondary cells, Journal of Power Sources 163 (2007) 1003-1039.

[3] L. Y. Beaulieu, K. W. Eberman, R. L. Turner, L. J. Krause, J. R. Dahn, Colossal reversible volume changes in lithium alloys, Electrochemical and Solid-State Letters 4 (2001) A137.

[4] C. K. Chan, H. Peng, G. Liu, K. McIlwrath, X. F. Zhang, R. A. Huggins, Y. Cui, High-performance lithium battery anodes using silicon nanowires, Nature Nanotechnology 3 (2008) 31-35.

[5] H. Wu, G. Chan, J. W. Choi, Y. Yao, M. T. McDowell, S. W. Lee, A. Jackson, Y. Yang, L. Hu, Y. Cui, Stable cycling of double-walled silicon nanotube battery anodes through solid-electrolyte interphase control, Nature nanotechnology 7 (5) (2012) $310-315$.

[6] N. Liu, H. Wu, M. T. McDowell, Y. Yao, C. Wang, Y. Cui, A yolk-shell design for stabilized and scalable li-ion battery alloy anodes, Nano letters 12 (6) (2012) 3315-3321.

[7] N. Liu, Z. Lu, J. Zhao, M. T. McDowell, H.-W. Lee, W. Zhao, Y. Cui, A pomegranate-inspired nanoscale design for large-volume-change lithium battery anodes, Nature nanotechnology 9 (3) (2014) 187-192.

[8] M. Ge, J. Rong, X. Fang, C. Zhou, Porous doped silicon nanowires for lithium ion battery anode with long cycle life, Nano letters 12 (5) (2012) 2318-2323.

[9] J. Zhu, C. Gladden, N. Liu, Y. Cui, X. Zhang, Nanoporous silicon networks as anodes for lithium ion batteries, Phys. Chem. Chem. Phys. 15 (2) (2012) 440-443.

[10] J. Y. Huang, L. Zhong, C. M. Wang, J. P. Sullivan, W. Xu, L. Q. Zhang, S. X. Mao, N. S. Hudak, X. H. Liu, A. Subramanian, H. Fan, L. Qi, A. Kushima, J. Li, In situ observation of the electrochemical lithiation of a single $\mathrm{SnO}_{2}$ nanowire electrode, Science 330 (2010) $1515-20$.

[11] X. H. Liu, L. Zhong, S. Huang, S. X. Mao, T. Zhu, J. Y. Huang, Size-dependent fracture of silicon nanoparticles during lithiation, ACS Nano 6 (2012) 1522-1531.

[12] M. T. McDowell, I. Ryu, S. W. Lee, C. Wang, W. D. Nix, Y. Cui, Studying the kinetics of crystalline silicon nanoparticle lithiation with in situ transmission electron microscopy, Advanced Materials 24 (2012) 6034-41.

[13] X. H. Liu, J. W. Wang, S. Huang, F. Fan, X. Huang, Y. Liu, S. Krylyuk, J. Yoo, S. A. Dayeh, A. V. Davydov, S. X. Mao, S. T. Picraux, S. Zhang, J. Li, T. Zhu, J. Y. Huang, In situ atomic-scale imaging of electrochemical lithiation in silicon, Nature Nanotechnology 7 (2012) 749-56.

[14] M. J. Chon, V. A. Sethuraman, A. McCormick, V. Srinivasan, P. R. Guduru, Real-time measurement of stress and damage evolution during initial lithiation of crystalline silicon, Physical Review Letters 107 (2011) 045503.

[15] X. H. Liu, H. Zheng, L. Zhong, S. Huang, K. Karki, L. Q. Zhang, Y. Liu, A. Kushima, W. T. Liang, J. W. Wang, J. H. Cho, E. Epstein, S. A. Dayeh, S. T. Picraux, T. Zhu, J. Li, J. P. Sullivan, J. Cumings, C. Wang, S. X. Mao, Z. Z. Ye, S. Zhang, J. Y. Huang, Anisotropic swelling and fracture of silicon nanowires during lithiation, Nano Letters 11 (2011) 3312-8.

[16] S. W. Lee, M. T. McDowell, L. A. Berla, W. D. Nix, Y. Cui, Fracture of crystalline silicon nanopillars during electrochemical lithium insertion, Proceedings of the National Academy of Sciences of the United States of America 109 (2012) 4080-5.

[17] J. W. Wang, Y. He, F. Fan, X. H. Liu, S. Xia, Y. Liu, C. T. Harris, H. Li, J. Y. Huang, S. X. Mao, T. Zhu, Two-phase electrochemical lithiation in amorphous silicon, Nano Letters 13 (2013) 709-15.

[18] M. T. McDowell, S. W. Lee, J. T. Harris, B. A. Korgel, C. Wang, W. D. Nix, Y. Cui, In situ TEM of two-phase lithiation of amorphous silicon nanospheres, Nano Letters 13 (2013) 758-64.

[19] H. Yang, F. Fan, W. Liang, X. Guo, T. Zhu, S. Zhang, A chemo-mechanical model of lithiation in silicon, Journal of the Mechanics and Physics of Solids 70 (2014) 349-361.

[20] S. Huang, F. Fan, J. Li, S. Zhang, T. Zhu, Stress generation during lithiation of high-capacity electrode particles in lithium ion batteries, Acta Materialia 61 (12) (2013) 4354-4364.

[21] J. W. Cahn, J. E. Hilliard, Free energy of a nonuniform system. I. Interfacial free energy, The Journal of Chemical Physics 28 (1958) 258.

[22] C. Di Leo, E. Rejovitzky, L. Anand, A Cahn-Hilliard-type phase-field theory for species diffusion coupled with large elastic deformations: Application to phase-separating Li-ion electrode materials, Journal of the Mechanics and Physics of Solids 70 (2014) 1-29. 
[23] C. Miehe, S. Mauthe, H. Ulmer, Formulation and numerical exploitation of mixed variational principles for coupled problems of CahnHilliard-type and standard diffusion in elastic solids, International Journal for Numerical Methods in Engineering 99 (2014) 737-762.

[24] K. Zhao, M. Pharr, Q. Wan, W. L. Wang, E. Kaxiras, J. J. Vlassak, Z. Suo, Concurrent reaction and plasticity during initial lithiation of crystalline silicon in lithium-ion batteries, Journal of the Electrochemical Society 159 (2012) A238-A243.

[25] Z. Cui, F. Gao, J. Qu, Interface-reaction controlled diffusion in binary solids with applications to lithiation of silicon in lithium-ion batteries, Journal of the Mechanics and Physics of Solids 61 (2013) 293-310.

[26] H. Larralde, M. Araujo, S. Havlin, H. Stanley, Reaction front for A+B-C diffusion-reaction systems with initially separated reactants, Physical Review A 46 (1992) 855-859.

[27] W. Wan, Q. Zhang, Y. Cui, E. Wang, First principles study of lithium insertion in bulk silicon, Journal of Physics: Condensed Matter 22 (2010) 415501.

[28] E. D. Cubuk, W. L. Wang, K. Zhao, J. J. Vlassak, Z. Suo, E. Kaxiras, Morphological evolution of Si nanowires upon lithiation: A firstprinciples multiscale model, Nano Letters 13 (5) (2013) 2011-2015.

[29] R. Grantab, V. B. Shenoy, Pressure-gradient dependent diffusion and crack propagation in lithiated silicon nanowires, Journal of The Electrochemical Society 159 (2012) A584.

[30] A. Khawam, D. R. Flanagan, Solid-state kinetic models: basics and mathematical fundamentals, The Journal of Physical Chemistry B 110 (2006) 17315-17328.

[31] L. Y. Lim, N. Liu, Y. Cui, M. F. Toney, Understanding phase transformation in crystalline Ge anodes for Li-ion batteries, Chemistry of Materials 26 (2014) 3739-3746.

[32] K. N. Tu, Selective growth of metal-rich silicide of near-noble metals, Applied Physics Letters 27 (1975) 221.

[33] N. Ding, J. Xu, Y. Yao, G. Wegner, X. Fang, C. Chen, I. Lieberwirth, Determination of the diffusion coefficient of lithium ions in nano-Si, Solid State Ionics 180 (2009) 222-225.

[34] G. A. Tritsaris, K. Zhao, O. U. Okeke, E. Kaxiras, Diffusion of Lithium in Bulk Amorphous Silicon: A Theoretical Study, The Journal of Physical Chemistry C 116 (2012) 22212-22216.

[35] E. Rabinowitch, Collision, co-ordination, diffusion and reaction velocity in condensed systems, Transactions of the Faraday Society 33 (1937) $1225-1233$.

[36] I. Ryu, J. W. Choi, Y. Cui, W. D. Nix, Size-dependent fracture of Si nanowire battery anodes, Journal of the Mechanics and Physics of Solids 59 (2011) 1717-1730.

[37] J. Ligenza, Effect of crystal orientation on oxidation rates of silicon in high pressure steam, The Journal of Physical Chemistry 65 (1961) 2011-2014.

[38] M. Stutzmann, The defect density in amorphous silicon, Philosophical Magazine Part B 60 (1989) 531-546.

[39] T. K. Bhandakkar, H. Gao, Cohesive modeling of crack nucleation under diffusion induced stresses in a thin strip: Implications on the critical size for flaw tolerant battery electrodes, International Journal of Solids and Structures 47 (10) (2010) 1424-1434.

[40] K. Zhao, Y. Li, L. Brassart, Pressure-sensitive plasticity of lithiated silicon in Li-ion batteries, Acta Mechanica Sinica 29 (2013) $379-387$.

[41] C. Linder, F. Armero, Finite elements with embedded strong discontinuities for the modeling of failure in solids, International Journal for Numerical Methods in Engineering 72 (2007) 1391-1433.

[42] F. Armero, C. Linder, New finite elements with embedded strong discontinuities in the finite deformation range, Computer Methods in Applied Mechanics and Engineering 197 (2008) 3138-3170.

[43] F. Armero, C. Linder, Numerical simulation of dynamic fracture using finite elements with embedded discontinuities, International Journal of Fracture 160 (2) (2009) 119-141.

[44] C. Linder, F. Armero, Finite elements with embedded branching, Finite Elements in Analysis and Design 45 (2009) $280-293$.

[45] C. Linder, D. Rosato, C. Miehe, New finite elements with embedded strong discontinuities for the modeling of failure in electromechanical coupled solids, Computer Methods in Applied Mechanics and Engineering 200 (2011) 141-161.

[46] C. Linder, An Analysis of the Exponential Electric Displacement Saturation Model in Fracturing Piezoelectric Ceramics, Technische Mechanik 32 (1) (2012) 53-69.

[47] C. Linder, C. Miehe, Effect of electric displacement saturation on the hysteretic behavior of ferroelectric ceramics and the initiation and propagation of cracks in piezoelectric ceramics, Journal of the Mechanics and Physics of Solids 60 (5) (2012) 882-903.

[48] C. Linder, A. Raina, A strong discontinuity approach on multiple levels to model solids at failure, Computer Methods in Applied Mechanics and Engineering 253 (2013) 558-583.

[49] C. Linder, X. Zhang, A marching cubes based failure surface propagation concept for three-dimensional finite elements with non-planar embedded strong discontinuities of higher-order kinematics, International Journal for Numerical Methods in Engineering 96 (2013) $339-372$.

[50] C. Linder, X. Zhang, Three-dimensional finite elements with embedded strong discontinuities to model failure in electromechanical coupled materials, Computer Methods in Applied Mechanics and Engineering 273 (2014) 143-160.

[51] A. Raina, C. Linder, A homogenization approach for nonwoven materials based on fiber undulations and reorientation, Journal of the Mechanics and Physics of Solids 65 (2014) 12-34.

[52] V. Schauer, C. Linder, All-electron Kohn-Sham density functional theory on hierarchic finite element spaces, Journal of Computational Physics 250 (2013) 644-664.

[53] V. Schauer, C. Linder, The reduced basis method in all-electron calculations with finite elements, Advances in Computational Mathematics (2014) 1-13 doi:10.1007/s10444-014-9374-z. 